\title{
A reusable localized surface plasmon resonance biosensor for quantitative detection of serum squamous cell carcinoma antigen in cervical cancer patients based on silver nanoparticles array
}

\author{
This article was published in the following Dove Press journal: \\ International Journal of Nanomedicine \\ 22 February 2014 \\ Number of times this article has been viewed
}

\section{Qianying Zhao \\ Ruiqi Duan' \\ Jialing Yuan' \\ Yi Quan' \\ Huan Yang ${ }^{2}$ \\ Mingrong $\mathrm{Xi}^{\prime}$}

'Department of Gynecology and Obstetrics, West China Second

University Hospital, Sichuan

University, ${ }^{2}$ State Key Laboratory of Optical Technologies for Microfabrication, Institute of Optics and Electronics, Chinese Academy of Science, Chengdu, Sichuan, People's Republic of China
Correspondence: Mingrong Xi Department of Gynecology and Obstetrics, West China Second University Hospital, Sichuan University, Chengdu, China. No 20, Block 3, Renminnan Road, Chengdu, Sichuan, People's Republic of China (61004I)

Tel +862885503348

Fax +862885502462

Email qiemingrong@163.com; qmrjzz@I26.com

\begin{abstract}
Squamous cell carcinoma antigen (SCCa), as a tumor biomarker, plays an important role in adjuvant diagnosis, treatment evaluation, and prognosis prediction for cervical cancer patients. Localized surface plasmon resonance (LSPR) technique based on noble metal nanoparticles bypasses the disadvantages of traditional testing strategies, in terms of free-labeling, short assay time, good sensitivity, and selectivity. Herein, we develop a novel and reusable LSPR biosensor for the detection of SCCa. First, a triangle-shaped silver nanoparticle array was fabricated using the nanosphere lithography method. Next, we investigated and verified the feasibility of amino coupling method using 11-mercaptoundecanoic acid (MUA) to form a functionalized chip surface with monoclonal anti-SCCa antibodies on the silver nanoparticles for distinct detection of SCCa. Different concentrations of SCCa were successfully tested in both buffer and human serum by the ultrasensitive and specific LSPR system, with a linear quantitative detection range of $0.1-1,000 \mathrm{pM}$ under optimal conditions. With appropriate regeneration solution, for example $50 \mathrm{mM}$ glycine- $\mathrm{HCl}$ ( $\mathrm{pH} 2.0$ ), the LSPR biosensor featured effective fabrication reproducibility, which reduced both production cost and testing time. Our study represents the first application of the LSPR biosensor in cervical cancer, and demonstrates that the rapid, simple, and reusable nanochip can serve as a potential alternative for clinical serological diagnosis of SCCa in cervical cancer patients.
\end{abstract}

Keywords: localized surface plasmon resonance, nanotechnology, biosensor, cervical cancer biomarker, squamous cell carcinoma antigen

\section{Introduction}

Cervical cancer is a public health problem worldwide. The disease ranks second in incidence, and is the fourth leading cause of cancer-related deaths among women worldwide. ${ }^{1,2}$ According to the International Federation of Gynecology and Obstetrics (FIGO), cervical cancer has a 5-year recurrence rate of $28 \%$ and a 5 -year overall mortality rate of $27.8 \%{ }^{3}$ The most common histological type of cervical cancer $(60 \%-80 \%)$ is squamous cell carcinoma (SCC). Squamous cell carcinoma antigen (SCCa), a glycoprotein with isoforms ranging from 45 to $55 \mathrm{kDa},{ }^{4}$ was first described as a tumor-associated antigen by Kato and Torigoe. ${ }^{5}$ Since it was first described, SCCa has been found to be elevated and proven helpful for the diagnosis and surveillance of various SCC of the uterine cervix, oral cavity, lung, skin, head and neck, esophagus, anal canal, and vulva. ${ }^{6-8}$ Currently, $\mathrm{SCCa}$, as a specific tumor marker, has been widely applied for the adjuvant diagnosis, prognostic risk evaluation, therapeutic monitoring, and follow-up of recurrence in patients 
with SCC of the uterine cervix. ${ }^{9}$ However, the clinical use of SCCa as a biomarker for early diagnosis of cervical cancer before treatment is severely restricted by the wide variation in detection sensitivity, ranging from $28 \%-88 \%$ according to different studies, due to various inclusion criteria, inconsistent cut-off values, and methodological defects. ${ }^{10}$

Enzyme linked immunosorbent assay (ELISA), radio immunoassay (RIA), and chemiluminescent immunoassay (CLIA) are often currently used for the detection of total SCCa in serum. Based on biological catalysis, ELISA shows good sensitivity in immunoassays, and is one of the most mature methods for protein detection. However, it still comes with shortcomings, such as pre-dilution of serum samples, narrow linear range, poor reproducibility, and long assay time. ${ }^{8}$ RIA methods, though considered accurate and reliable, have constraints for clinical application due to radioisotopes and short half-life, waste disposal problems, and the need for specialized laboratories. CLIA, an automatic and radiation-free technique, also has drawbacks, including the large volume of the analysis instrument, high cost, and special labeling requirements. ${ }^{11}$ These limitations listed above have induced an intensive search for an alternative technique that is sensitive, specific, rapid, simple, low-cost, and environmentally friendly.

Recently, biosensors based on noble metal nanoparticles (eg, silver or gold) have attracted tremendous research attention because of their unique optical and electrical properties. Localized surface plasmon resonance (LSPR), a specific characteristic of metallic or metalized nanostructure materials, is generated when the incident photon frequency resonates with the collective oscillation of free electrons. ${ }^{12-14}$ The LSPR extinction spectrum, which can be monitored in the ultraviolet (UV)-visible region, is known to be associated with the composition, size, shape, orientation, and local dielectric environment of nanoparticles. ${ }^{14,15}$ In particular, the peak wavelength of the LSPR extinction spectrum $\left(\lambda_{\max }\right)$ is highly sensitive to even subtle changes of the local refractive index near the nanoparticle surface induced by bio-molecular interactions. ${ }^{16}$ This optical property enables noble metal nanoparticles to serve as biosensors that can transform biological recognition information into analytically useful signals in the form of LSPR $\lambda_{\text {max }}$ shifts. The applicability of this biosensor has been demonstrated in a wide range of fields, such as drug screening, medical diagnosis, food safety, and environmental monitoring. ${ }^{13,14,17}$ Moreover, the LSPR biosensor has significant advantages in the form of label-free biomarker detection, rapid test time, and direct assay format, unlike the traditional immunoassay approaches (eg, ELISA); and compared with chemiluminescent analysis and current commercial surface plasmon resonance sensors, the LSPR sensor has more outstanding features, including miniaturization, portability, and low cost. ${ }^{11}$

In the present study, we developed an LSPR biosensor, based on the triangular silver (Ag) nanoparticles array, with monoclonal anti-SCCa antibodies immobilized on the chip for direct detection of SCCa in blood samples from patients with cervical cancer. Next, SCCa in both buffer and human serum samples were tested under optimum conditions. Further, the performance (detection limit, linear range, selectivity, regeneration, and stability) of the sensor was analyzed and discussed in detail. To the best of our knowledge, ours is the first study to investigate the applicability of the LSPR technique for the detection of SCCa in either buffer or serum.

\section{Materials and methods Materials}

11-Mercaptoundecanoic acid (MUA), N-hydroxysuccinimide (NHS), phosphate-buffered saline (PBS; $10 \mathrm{mM}, \mathrm{pH} 7.4$ ), and bovine serum albumin (BSA) were purchased from Sigma-Aldrich (St Louis, MO, USA). 1-Ethyl-3-(3-dimethylaminopropyl) carbodiimide hydrochloride (EDC) was purchased from Aladdin (Shanghai, People's Republic of China). Mouse monoclonal anti-SCCa antibody (anti-SCCa) and standard SCCa were obtained from Origene Technologies Inc., (Rockville, MD, USA). Ethanolamine, absolute ethanol, glycine, sodium hydroxide $(\mathrm{NaOH})$, hydrochloric acid $(\mathrm{HCl})$, urea, and Tween-20 were purchased from the Kelong Company (Chengdu, People's Republic of China). Ultrapure water $(18.3 \mathrm{M} \Omega / \mathrm{cm})$ obtained from EMD Millipore Corporation (Bellerica, MA, USA) was used for preparation of all solutions. All reagents used were of analytical reagent grade.

\section{Patients and samples}

The human serum samples from cervical cancer patients as well as controls were collected from West China Second University Hospital (Chengdu, People's Republic of China). Written informed consent was not obtained; all specimens were leftover blood samples from routine blood tests, thus causing no extra discomfort to the participants. Sera were isolated from whole blood samples via centrifugation at 3,000 rpm for 15 minutes, and subsequently kept frozen at $-80^{\circ} \mathrm{C}$ until analysis.

\section{Construction of LSPR biosensor and observation process}

The integrated LSPR nanosensor was built on-site, as previously described in detail. ${ }^{18}$ Briefly, nanosphere 
lithography (NSL) technology was used to fabricate the sensor chip based on triangular-shaped silver nanoparticles array.

The peak wavelength of the LSPR extinction spectrum $\left(\lambda_{\max }\right)$ excited by the silver nanoparticles was measured and recorded by a UV-visible spectrometer (Model 9055; Sciencetech Corp, Ottawa, ON, Canada). Specifically, white light emerging from the optical fiber bundle transmitted through a collimating lens, providing the incident light. When the nanochip was placed perpendicular to the incident light, the distinct LSPR extinction spectra could be excited at room temperature, directly obtained by the spectroscope ranging from $400 \mathrm{~nm}$ to $800 \mathrm{~nm}$, and immediately displayed on the computer screen.

All the extinction spectra were then recorded and analyzed through Spectra Suite software (Ocean Optics, Dunedin, FL, USA). A shift toward the longer wavelength region was referred to as a red-shift and indicated by $(+)$; whereas a shift toward the shorter wavelength region was defined as a blue-shift and denoted as (-). The relative wavelength shift, namely $\Delta \lambda_{\max }$, was used to monitor the binding of target analytes. ${ }^{19}$

\section{Functionalization of the LSPR biosensor}

The sensor chip was functionalized through multiple steps for specific detection of bio-molecular interaction. To form a self-assembled monolayer (SAM) on the slice surface, the silver nanochip was firstly immersed in $1 \mathrm{mM}$ MUA solution (in ethanol) for 10 hours at room temperature, then washed thoroughly with pure ethanol and dried at room temperature. Subsequently, the sensor was incubated in $75 \mathrm{mM}$ EDC$\mathrm{HCl} / 15 \mathrm{mM}$ NHS (volume/volume =1) solution for another hour at room temperature to activate the carboxyl group of the SAM, which could react with amino groups provided by antibodies to form amides. In this step, EDC, a zero-length coupling agent, coupled the amine groups on the antibody to the carboxyl groups on MUA. Next, $60 \mu \mathrm{L}$ diluted anti-SCCa monoclonal antibody solution (in PBS, $\mathrm{pH} 7.4$ ) at $10 \mu \mathrm{g} / \mathrm{mL}$ was spotted on the modified chip surface and overnight incubation in a humidified chamber at $4{ }^{\circ} \mathrm{C}$ followed. Finally, the chip was immersed in $0.5 \mathrm{M}$ ethanolamine- $\mathrm{HCl}$ solution $(\mathrm{pH}$ 8.5 ) for 30 minutes to deactivate the unreacted esters, after which the slice was rinsed with ultrapure water and dried at room temperature. The immobilization process is outlined in Figure 1.

\section{Detection of SCCa by LSPR biosensor and performance assessment}

As shown in Figure 1E, after the immobilization of antibody onto the chips, both commercial SCCa solution at various concentrations and serum samples were introduced to the

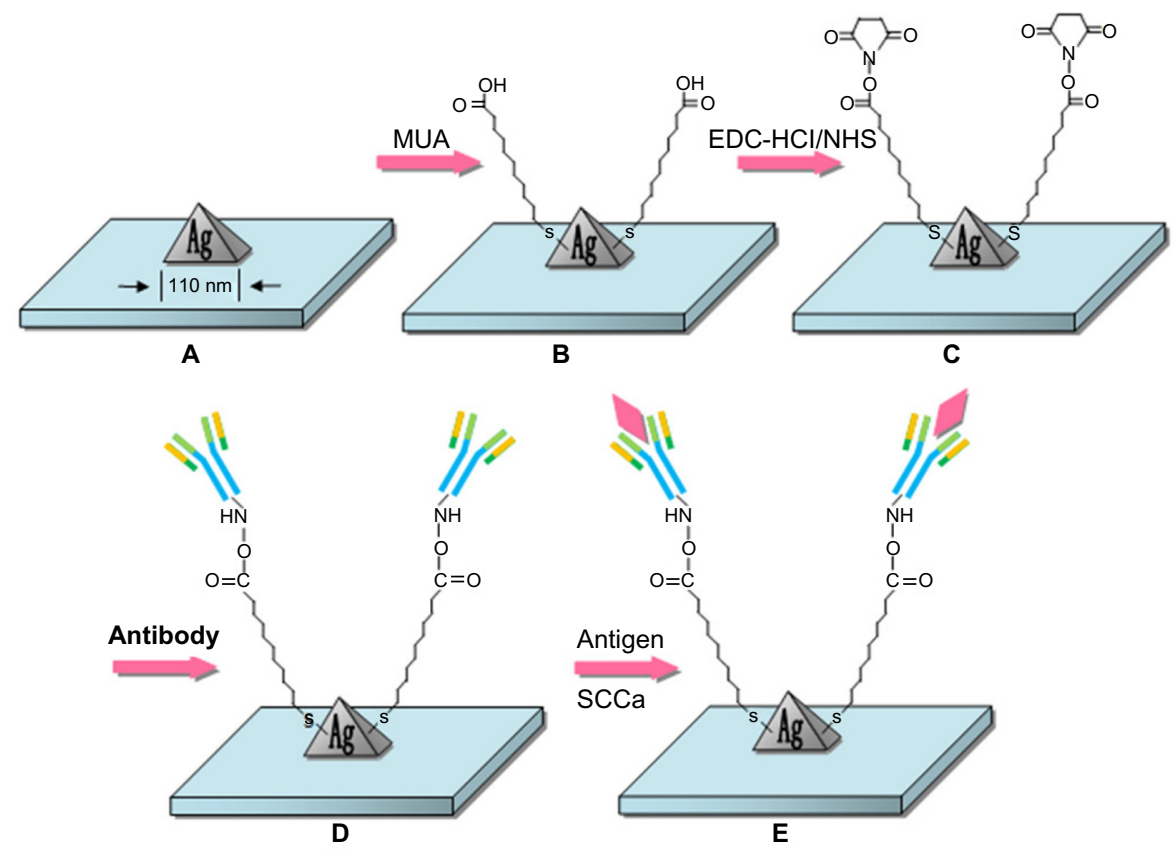

Figure I Design of the LSPR biosensor for specific detection of SCCa. (A) Glass substrate with triangle-shaped silver nanoparticles array synthesized by NSL technology. (B) SAM formed by incubation of I mM MUA. (C) Incubation in $75 \mathrm{mM}$ EDC-HCl/I5 mM NHS. (D) Attachment of anti-SCCa antibody (I0 $\mu \mathrm{g} / \mathrm{mL}$ ). (E) Immunoassay of SCCa in both buffer and serum samples.

Abbreviations: Ag, silver; EDC, I-Ethyl-3-(3-dimethylaminopropyl) carbodiimide hydrochloride; LSPR, localized surface plasmon resonance; MUA, I I-mercaptoundecanoic acid; NHS, N-hydroxysuccinimide; NSL, nanosphere lithography; SAM, self-assembled monolayer; SCCa, squamous cell carcinoma antigen. 
biosensor and incubated at room temperature for 30 minutes. The chips were dried after thorough rinsing with $\mathrm{PBS}(0.05 \%$ Tween-20) and subsequently with ultrapure water. Next, the change in LSPR extinction spectrum caused by antibodyantigen combination was observed and recorded. Performance of the LSPR biosensor, including limit of detection, linear range, specificity, precision and regeneration capability, were analyzed and evaluated using a software program (Origin 7.5; OriginLab Corporation, Northampton, MA, USA).

\section{Results and discussion Immobilization process and spectroscopy observation}

The LSPR response to the immobilization process and detection of SCCa are shown in Figure 2. It is well known that molecular binding onto the nanochip surface can induce an increase in the local refractive index around nanoparticles, thus resulting in a red-shift of LSPR spectrum peak position denoted as peak wavelength, $\lambda_{\max }{ }^{13,14,20}$ Therefore, $\Delta \lambda_{\max }$ can act as a reliable indicator of a bioreaction near nanoparticles. A spectrometer was used to monitor the LSPR extinction spectrum in each step. Before modification, the LSPR $\lambda_{\text {max }}$ of the bare silver nanochip was $573.92 \mathrm{~nm}$ as depicted in Figure 2A. After modification of MUA onto the silver surface, the LSPR $\lambda_{\text {max }}$ shifted to 589.1 (Figure 2B) with a corresponding $\Delta \lambda_{\text {max }}$ of $+15.18 \mathrm{~nm}$. Additional red-shift $(+13.28 \mathrm{~nm})$ of LSPR $\lambda_{\text {max }}$ was observed after incubation of anti-SCCa monoclonal antibodies onto the sensor when

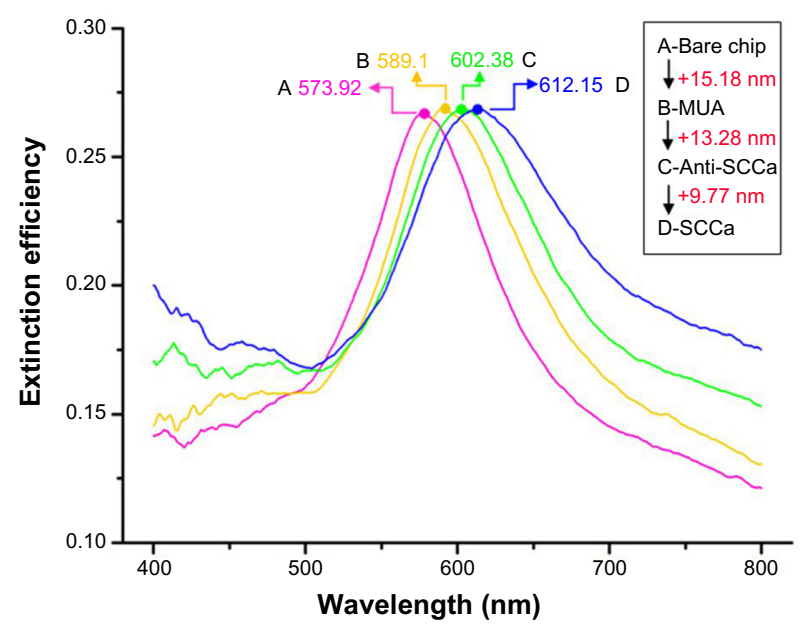

Figure 2 LSPR spectra for processing steps of immobilization and detection of $100 \mathrm{pM}$ SCCa. (A) Bare silver nanochip, $\lambda_{\max }=573.92 \mathrm{~nm}$. (B) Modification of I mM MUA, $\lambda_{\max }=589.1 \mathrm{~nm}$. (C) Incubation with $10 \mu \mathrm{g} / \mathrm{mL}$ monoclonal anti-SCCa, $\lambda_{\max }=602.38 \mathrm{~nm}$. (D) Detection of $100 \mathrm{pm} \mathrm{SCCa}, \lambda_{\max }=612.15 \mathrm{~nm}$.

Note: All spectra were collected at room temperature in air.

Abbreviations: LSPR, localized surface plasmon resonance; MUA, I I-mercaptoundecanoic acid; SCCa, squamous cell carcinoma antigen. the LSPR $\lambda_{\max }$ was measured at $602.38 \mathrm{~nm}$ (Figure 2C). Finally, introduction of 100 pM SCCa caused another $\Delta \lambda_{\text {max }}$ of $+9.77 \mathrm{~nm}$, achieving a representative LSPR $\lambda_{\text {max }}$ of $612.15 \mathrm{~nm}$ (Figure 2D).

The statistics showed potential serum sample detection with the LSPR biosensor, as standard SCCa in buffer was successfully detected. Furthermore, combined with our previous work, ${ }^{11,21}$ this validates that the amino-coupling method based on MUA and EDC/NHS is feasible and effective for connecting antibodies to noble metal material. Also, the modified fabrication procedures, as shown in Figure 1, could serve as a general process for biological-sensitive layer construction.

\section{Selectivity test}

Selectivity is one of the most fundamental properties that a sensor should acquire. Therefore, several control experiments were conducted to ensure that the results were not disturbed by nonspecific bindings. In order to achieve specific detection, $0.5 \mathrm{M}$ ethanolamine- $\mathrm{HCl}$ solution $(\mathrm{pH} 8.5)$ was introduced as a surface passivation agent after sufficient immobilization of antibodies. The resolution of our system was $3 \mathrm{~nm}$ corresponding to a signal-to-noise ratio of three, given that the noise $(0.995 \mathrm{~nm})$ was calculated as the standard deviation of the blank controls $(n=10)$. This indicates that a target molecule being sensed at the nanochip interface when $\Delta \lambda_{\text {max }}>3 \mathrm{~nm}$ or $\Delta \lambda_{\max }<3 \mathrm{~nm}$ could be attributed to the inevitable detection bias of the system.

Human epididymis secretory protein 4 (HE4), another tumor marker, and bovine serum albumin (BSA), the most abundant protein component in blood plasma were chosen as interferences. BSA has also been heralded as a potential interfering bio-molecule, especially when it comes to detection of low-abundant proteins in biological fluids. ${ }^{22}$ The functionalized biosensor was incubated in 100 pM HE4 or BSA for 30 minutes at room temperature, and all control experiments were implemented in triplicate. Results show that there was hardly any shift in the LSPR peak $\left(\Delta \lambda_{\max }<3 \mathrm{~nm}\right)$ after PBS rinsing, which indicates excellent selectivity of the detection system based on highly specific antibody-antigen reaction (Figure 3).

\section{Precision evaluation}

The stability within and between batches is an important factor to ensure the reliability of test results in practical application. ${ }^{23}$ Precision was evaluated by the variation coefficients (CVs) of intra- and inter-assays at two SCCa 

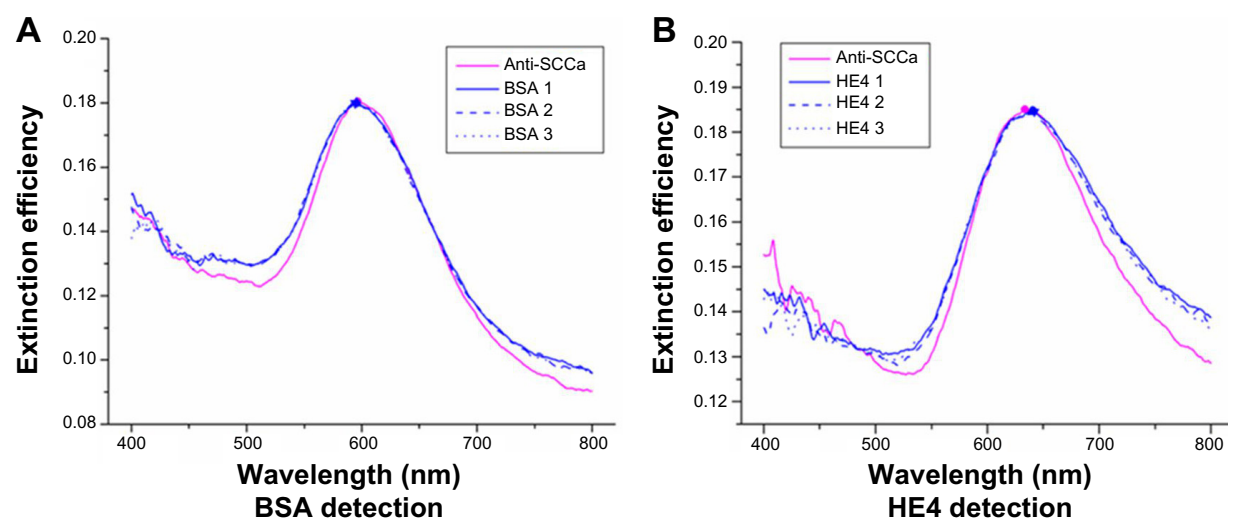

Figure 3 Selectivity test of the LSPR biosensor. (A) Detection of $100 \mathrm{pM} \mathrm{BSA}, \Delta \lambda_{\max }=-0.8 \mathrm{I} \mathrm{nm},+0.4 \mathrm{Imm},+1.03 \mathrm{~nm}$. (B) Detection of I00 pM HE4, $\Delta \lambda_{\max }=+\mathrm{I} .2 \mathrm{~nm}$, $+0.20 \mathrm{~nm},+1.00 \mathrm{~nm}$.

Note: All control experiments were completed in triplicate.

Abbreviations: BSA, bovine serum albumin; HE4, human epididymis secretory protein 4; LSPR, localized surface plasmon resonance; SCCa, squamous cell carcinoma antigen.

concentrations (10 pM and $100 \mathrm{pM})$. The intra-batch $\mathrm{CV}$ of the proposed sensor was calculated by three successive measurements using chips of the same batch. Similarly, interrun precision was tested independently with three biosensors randomly selected from three batches. The results, as shown in Table 1, suggest that the immunosensor showed acceptable precision and is suitable for application.

\section{Regeneration and reproductivity}

After completion of the antigen-antibody interaction, SCCa can be removed using regeneration solution, making the functionalized chip surface reusable. Ideally, any binding of SCCa analyte should be removed while the activity of anti-SCCa molecules should not be disturbed. To evaluate the performance of the regeneration process, the residual activity of immobilized anti-SCCa was calculated as the numerical value change of detection results $\left(\Delta \lambda_{\max } \%\right)$, defined as follows:

$$
\Delta \lambda_{\max } \%=\left(\Delta \lambda_{\max }\right)_{\text {post }} /\left(\Delta \lambda_{\max }\right)_{\text {pre }} \times 100 \%
$$

where $\left(\Delta \lambda_{\text {max }}\right)_{\text {post }}$ is the post-regeneration detection result and $\left(\Delta \lambda_{\max }\right)_{\text {pre }}$ is the pre-regeneration detection result.

Table I Precision test for the LSPR biosensor

\begin{tabular}{|c|c|c|c|c|}
\hline $\begin{array}{l}\mathrm{SCCa} \\
\text { concentration } \\
\text { (pM) }\end{array}$ & $\begin{array}{l}\text { Intra-assay } \\
\bar{x} \pm S D \\
(n=3, n m)\end{array}$ & CV (\%) & $\begin{array}{l}\text { Inter-assay } \\
\bar{x} \pm S D \\
(\mathrm{n}=3, \mathrm{~nm})\end{array}$ & CV (\%) \\
\hline 10 & $6.98 \pm 0.28$ & 4.01 & $7.16 \pm 0.24$ & 3.35 \\
\hline 100 & $9.66 \pm 0.32$ & 3.31 & $9.76 \pm 0.45$ & 4.61 \\
\hline
\end{tabular}

Notes: $\bar{x}$ is mean of test results; $C V(\%)=S D / \bar{x}$.

Abbreviations: LSPR, localized surface plasmon resonance; SCCa, squamous cell carcinoma antigen; SD, standard deviation; CV variation coefficient.
If post-regeneration binding remains above $90 \%$ compared to the binding efficiency before regeneration, the experiment conditions should be considered adequate. ${ }^{24}$ Different types of regenerating agents from three common categories (high ionic strength, low $\mathrm{pH}$, high $\mathrm{pH}$ ) were tested at least three times and average residual detection effectiveness is shown in Table 2 . The results indicate that $50 \mathrm{mM}$ glycine- $\mathrm{HCl}$ buffer $(\mathrm{pH} 2.0)$ is effective and optimal for regeneration with residual activity of $94.86 \% \pm 4.47 \%$, compared to $0.01 \mathrm{M}$ $\mathrm{NaOH}$ and 6.0 M urea.

The regeneration process was done by immersing chips into $50 \mathrm{mM}$ glycine- $\mathrm{HCl}(\mathrm{pH} 2.0)$ for about 3 minutes and rinsing with PBS thereafter. The reproducibility also proved feasible at other SCCa concentrations (100 pM and 1,000 pM) with both identical and different biosensors. The results in Table 3 show that the LSPR shift remained more than $90 \%$ of the initial response after performing regeneration four times, demonstrating that the modified sensors were reusable and able to provide reliable detection outcomes. However, the LSPR $\Delta \lambda_{\text {max }}$ value dropped below $90 \%$ of the original signal after regenerating more than five times, which may be caused

Table 2 Efficiency of different regeneration agents

\begin{tabular}{|c|c|c|}
\hline \multirow[t]{2}{*}{$\begin{array}{l}\text { Regeneration } \\
\text { solution }\end{array}$} & \multicolumn{2}{|c|}{$\begin{array}{l}\text { Residual detection } \\
\text { effectiveness }^{\mathrm{a}}\left(\Delta \lambda_{\max } \%\right)\end{array}$} \\
\hline & $\bar{x} \pm \operatorname{SD}(n \geq 3, \%)$ & CV (\%) \\
\hline $0.01 \mathrm{M} \mathrm{NaOH}$ & $79.16 \pm 24.44$ & 30.87 \\
\hline 6.0 M urea & $76.19 \pm 19.24$ & 25.25 \\
\hline 50 mM glycine- $\mathrm{HCl}(\mathrm{pH} 2.0)$ & $94.86 \pm 4.47$ & 4.71 \\
\hline
\end{tabular}


Table 3 Reusability of the LSPR biosensor

\begin{tabular}{|c|c|c|c|c|c|c|c|c|}
\hline \multirow{2}{*}{$\begin{array}{l}\text { Sensor } \\
\text { code }\end{array}$} & \multirow{2}{*}{$\begin{array}{l}\text { Concentration } \\
\text { (pM) }\end{array}$} & \multicolumn{5}{|c|}{ LSPR shift $\left(\Delta \lambda_{\max }\right)$} & \multirow{2}{*}{$\begin{array}{l}\bar{x} \pm S D \\
(n=5, n m)\end{array}$} & \multirow[t]{2}{*}{ CV (\%) } \\
\hline & & 01 & 02 & 03 & 04 & 05 & & \\
\hline $0 \mathrm{I}$ & 100 pM & 9.89 & 9.72 & 9.71 & 9.30 & 8.97 & $9.52 \pm 0.38$ & 3.99 \\
\hline 02 & $\mathrm{I}, 000 \mathrm{pM}$ & 12.92 & 12.14 & 12.37 & 11.63 & 11.53 & $12.12 \pm 0.59$ & 4.87 \\
\hline
\end{tabular}

Notes: $\bar{x}$ is the mean of LSPR shifts obtained from initial detection and following regeneration procedures; $C V(\%)=S D / \bar{x}$.

Abbreviations: LSPR, localized surface plasmon resonance; SD, standard deviation; CV variation coefficient; $\Delta \lambda_{\text {max }}$, peak wavelength shift/variation of LSPR spectrum.

by oxidation of the silver surface or damaged anti-SCCa reactivity. We attempted to identify the underlying causes for limited reproductive times by scanning electron microscopy and atomic force microscopy; however, no significant imaging findings were gained.

\section{Calibration curve}

The biosensors were incubated in different concentrations of standard SCCa solution ranging from $0.1 \mathrm{pM}$ to $10,000 \mathrm{pM}$ under optimal conditions, and LSPR extinction spectra were obtained with peak shifts recorded. Mean value and standard deviation of $\Delta \lambda_{\text {max }}$ were calculated, since each concentration measurement was repeated three times. As shown in Figure 4, the LSPR $\Delta \lambda_{\text {max }}$ values increased stepwise with increasing SCCa concentrations and were linear with the logarithm of concentrations within $0.1 \mathrm{pM}$ to $1,000 \mathrm{pM}$ (Figure 4), which is a broader range than that of the commercial ELISA kit (1.75 pM to $115 \mathrm{pM})$. In addition, the linear regression equation was:

$$
\operatorname{LSPR}(\mathrm{nm})=2.29 \times \lg [\mathrm{SCCa}](\mathrm{pM})+5.07
$$

with a linear correlation coefficient $(R)$ of 0.996 . Based on the system resolution $(3 \mathrm{~nm})$ described previously, the estimated

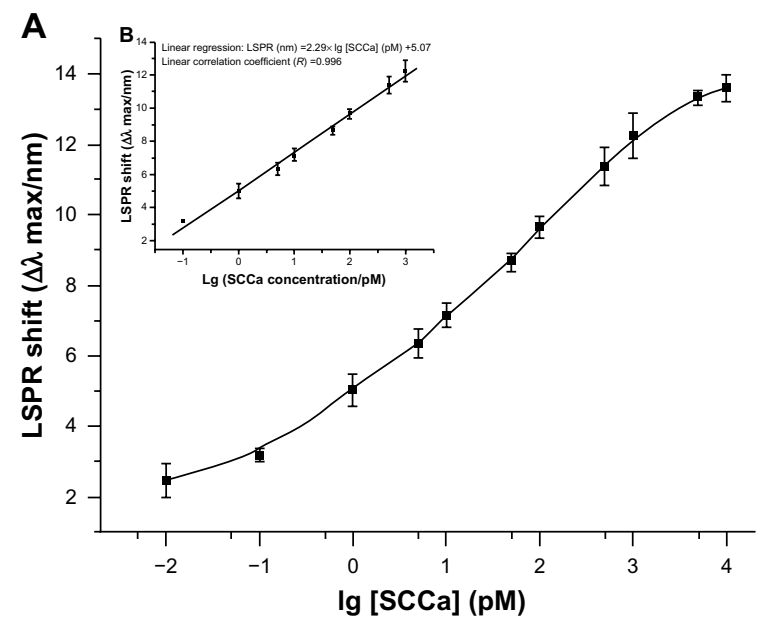

Figure 4 LSPR calibration curves. (A) Calibration curve of LSPR shift versus the logarithm of SCCa concentration. (B) The linear relationship between the LSPR shift and the logarithm of SCCa concentration in the range from $0.1 \mathrm{pM}$ to $1,000 \mathrm{pM}$.

Abbreviations: LSPR, localized surface plasmon resonance; SCCa, squamous cell carcinoma antigen; Ig, logarithm. limit of detection (LOD) defined as the analyte concentration corresponding to a signal-to-noise ratio of three was calculated to be $0.125 \mathrm{pM}$. This is comparable to the routine CLIA method $(0.25 \mathrm{pM})$ and more than sufficient for analysis of SCCa in serum where normal SCCa values are considered to be less than $45 \mathrm{pM} .{ }^{25}$ In general, the LSPR biosensor had good analytical performance and showed potential for blood-sample detection without pre-dilution or radio-labeling.

\section{Serum sample detection}

This study also investigated the practicability of the proposed LSPR sensor for detecting SCCa in human serum. SCCa concentration of each serum sample was calculated according to the linear regression equation. A series of ten serum samples from cervical cancer patients (FIGO staging Ia-IIa) were analyzed. The average SCCa level in this group was found to be $56.31 \mathrm{pM}$, corresponding to a mean LSPR shift of $8.95 \mathrm{~nm}$, which was more than $45 \mathrm{pM}$ - the cutoff value with $88 \%$ specificity. ${ }^{23}$ The control group included another ten serum specimens from patients with other gynecological diseases, such as myoma of the uterus, ovarian tumors, adenomyosis of the uterus, and so on. Although the concentrations of SCCa ranging from 21.47 $\mathrm{pM}$ to $124.76 \mathrm{pM}$ in the cancer group covered the cutoff value $(45 \mathrm{pM})$, they were, based on a Student's $t$-test, significantly $(P<0.05)$ higher than those of the control group (Table 4).

Red shifts less than $4.88 \mathrm{~nm}$ were observed in the controls, validating that the biosensor had good specificity and the results were reliable without disruption of other components in the serum. Moreover, the SCCa levels of the serum samples were within the linear range of the LSPR sensor. However, the sensitivity of SCCa using the LSPR system compared to pathological diagnosis was only $50 \%$ due to the limited

Table 4 Test of serum samples by LSPR biosensor

\begin{tabular}{llc}
\hline Group & LSPR shift $\left(\Delta \lambda_{\max }\right)$ & SCCa concentration \\
& $\overline{\mathbf{x}} \pm \mathbf{S D}(\mathbf{n}=\mathbf{1 0}, \mathbf{n m})$ & $\overline{\mathbf{x}} \pm \mathbf{S D}(\mathbf{n}=1 \mathbf{0}, \mathbf{p M})$ \\
\hline Cancer group & $8.95 \pm 0.53$ & $56.311 \pm 31.89$ \\
Control group & $3.12 \pm 1.16$ & $0.24 \pm 0.25$ \\
\hline
\end{tabular}

Note: $\bar{x}$ is the mean of test results.

Abbreviations: LSPR, localized surface plasmon resonance; SCCa, squamous cell carcinoma antigen; SD, standard deviation; $\Delta \lambda_{\text {max }}$, peak wavelength shift/variation of LSPR spectrum. 
number of subjects, indicating the necessity of large samplesize clinical research. Aside from these data, the LSPR sensor test needs to be validated by traditional methods (eg, ELISA). Also, the precision of quantitative analysis needs improvement with larger shifts from the same amount of analyte.

\section{Conclusion}

To the best of our knowledge, this is the first use of the LSPR method for medical diagnosis in cervical cancer patients. The experiments described in this study demonstrate that: 1) a self-assembled MUA monolayer is suitable linkage for the effective immobilization of anti-SCCa onto the silver nanochips via amino coupling; 2) the functionalized LSPR sensor is simple to prepare, can provide good sensitivity, has a low detection limit, and has good selectivity for assaying SCCa; 3) using an appropriate regeneration solution, the sensor can be reused with good signal retention, which is both time and cost efficient; 4) the custom-built LSPR system is available for quantitative analysis of SCCa level in human serum with advantages in terms of a rapid test time, label-free, and dilution-free process. Further studies need to be performed to evaluate the applicability of this biosensor in medical diagnosis for gynecological malignancies with a large, blinded diagnostic trial compared to the gold standard method. Signal amplification systems should be explored in order to lower the magnitude of the detecting sensitivity, as well as improving the quantitative measurement accuracy. ${ }^{13}$ In conclusion, the LSPR biosensor is a promising platform for cancer biomarker studies and commercial application.

\section{Acknowledgments}

This project was financially supported by National Key Basic Research Program of China (2011CB301800) and Sichuan Provincial Science and Technology Support Project (2012SZ0021). The authors thank Qianqian Gao and Lin Li for their kind contribution to this work.

\section{Disclosure}

The authors report no conflicts of interest in this work.

\section{References}

1. Li J, Li LK, Ma JF, et al. Knowledge and attitudes about human papillomavirus (HPV) and HPV vaccines among women living in metropolitan and rural regions of China. Vaccine. 2009;27(8):1210-1215.

2. Woodman CB, Collins SI, Young LS. The natural history of cervical HPV infection: unresolved issues. Nat Rev Cancer. 2007;7(1): $11-22$.

3. Muñoz N, Bosch FX, de Sanjosé S, et al; International Agency for Research on Cancer Multicenter Cervical Cancer Study Group. Epidemiologic classification of human papillomavirus types associated with cervical cancer. $N$ Engl J Med. 2003;348(6):518-527.
4. Kato H, Morioka H, Aramaki S, Torigoe T. Radioimmunoassay for tumor-antigen of human cervical squamous cell carcinoma. Cell Mol Biol Incl Cyto Enzymol. 1979;25(1):51-56.

5. Kato H, Torigoe T. Radioimmunoassay for tumor antigen of human cervical squamous cell carcinoma. Cancer. 1977;40(4): $1621-1628$.

6. Ho YJ, Hsieh JF, Tasi SC, Lee JK, Kao CH. Tissue polypeptide specific antigen and squamous cell carcinoma antigen for early prediction of recurrence in lung squamous cell carcinoma. Lung. 2000;178(2): $75-80$.

7. Bandoh N, Ogino T, Katayama A, et al. HLA class I antigen and transporter associated with antigen processing downregulation in metastatic lesions of head and neck squamous cell carcinoma as a marker of poor prognosis. Oncol Rep. 2010;23(4):933-939.

8. Zhang H, Qi S. A rapid and sensitive chemiluminescence immunoassay based on magnetic particles for squamous cell carcinoma antigen in human serum. Clin Chim Acta. 2011;412(17-18): $1572-1577$

9. Zhao Q, Feng Y, Mao X, Qie M. Prognostic value of fluorine-18fluorodeoxyglucose positron emission tomography or PET-computed tomography in cervical cancer: a meta-analysis. Int J Gynecol Cancer. 2013;23(7):1184-1190.

10. Gadducci A, Tana R, Cosio S, Genazzani AR. The serum assay of tumour markers in the prognostic evaluation, treatment monitoring and follow-up of patients with cervical cancer: a review of the literature. Crit Rev Oncol Hematol. 2008;66(1):10-20.

11. Yuan J, Duan R, Yang H, Luo X, Xi M. Detection of serum human epididymis secretory protein 4 in patients with ovarian cancer using a label-free biosensor based on localized surface plasmon resonance. Int J Nanomedicine. 2012;7:2921-2928.

12. Zhao J, Zhang X, Yonzon CR, Haes AJ, Van Duyne RP. Localized surface plasmon resonance biosensors. Nanomedicine (Lond). 2006;1(2):219-228.

13. Bingham JM, Hall WP, Van Duyne RP. Exploring the unique characteristics of LSPR biosensing. In: Dmitriev A, editor. Nanoplasmonic Sensors. New York: Springer; 2012:29-58.

14. Petryayeva E, Krull UJ. Localized surface plasmon resonance: nanostructures, bioassays and biosensing - a review. Anal Chim Acta. 2011;706(1):8-24.

15. Anker JN, Hall WP, Lyandres O, Shah NC, Zhao J, Van Duyne RP. Biosensing with plasmonic nanosensors. Nat Mater. 2008;7(6): $442-453$.

16. Monk DJ, Walt DR. Optical fiber-based biosensors. Anal Bioanal Chem. 2004;379(7-8):931-945.

17. Kreuzer MP, Quidant R, Salvador JP, Marco MP, Badenes G Colloidal-based localized surface plasmon resonance (LSPR) biosensor for the quantitative determination of stanozolol. Anal Bioanal Chem. 2008;391(5):1813-1820.

18. Zhu SL, Li F, Du CL, Fu YQ. A localized surface plasmon resonance nanosensor based on rhombic Ag nanoparticle array. Sens Actuators B Chem. 2008;134:193-198.

19. Haes AJ, Hall WP, Chang L, Klein WL, Van Duyne RP. A localized surface plasmon resonance biosensor: first steps toward an assay for Alzheimer's disease. Nano Lett. 2004;4:1029-1034.

20. Duan RQ, Yuan JL, Yang H, Luo XG, Xi MR. Detection of p53 gene mutation by using a novel biosensor based on localized surface plasmon resonance. Neoplasma. 2012;59(3):348-353.

21. Lai T, Hou Q, Yang H, Luo X, Xi M. Clinical application of a novel sliver nanoparticles biosensor based on localized surface plasmon resonance for detecting the microalbuminuria. Acta Biochim Biophys Sin (Shanghai). 2010;42(11):787-792.

22. Vestergaard M, Kerman K, Kim DK, Ha MH, Tamiya E. Detection of Alzheimer's tau protein using localised surface plasmon resonancebased immunochip. Talanta. 2008;74(4):1038-1042.

23. Chalquest RR. Quantitation of indirect sandwich enzyme-linked immunosorbent assay parameters. J Clin Microbiol. 1988;26(1): $147-148$. 
24. Van der Merwe PA. Surface plasmon resonance. In: Harding SE, Chowdhry BZ, editors. Protein-ligand Interactions: Hydrodynamics and Calorimetry: a Practical Approach. New York: Oxford University Press; 2000:828.
25. Gadducci A, Tana R, Cosio S, Genazzani AR. The serum assay of tumour markers in the prognostic evaluation, treatment monitoring and follow-up of patients with cervical cancer: a review of the literature. Crit Rev Oncol Hematol. 2008;66(1):10-20.

\section{Publish your work in this journal}

The International Journal of Nanomedicine is an international, peerreviewed journal focusing on the application of nanotechnology in diagnostics, therapeutics, and drug delivery systems throughout the biomedical field. This journal is indexed on PubMed Central, MedLine, CAS, SciSearch $\AA$, Current Contents ${ }^{\circledR} /$ Clinical Medicine,
Journal Citation Reports/Science Edition, EMBase, Scopus and the Elsevier Bibliographic databases. The manuscript management system is completely online and includes a very quick and fair peer-review system, which is all easy to use. Visit http://www.dovepress.com/ testimonials.php to read real quotes from published authors. 